\title{
Channel Load Aware Routing in Wireless Mesh Networks
}

\author{
Nemesio A. Macabale Jr.*, Roel M. Ocampo ${ }^{\dagger}$, Cedric Angelo M. Festin ${ }^{\star}$ \\ *Department of Information Technology, Central Luzon State University, Science City of Muñoz, Philippines \\ ${ }^{\dagger}$ Electrical and Electronics Engineering Institute, University of the Philippines, Quezon City, Philippines \\ ${ }^{\ddagger}$ Department of Computer Science, University of the Philippines, Quezon City, Philippines \\ namacabale@clsu.edu.ph, roel@upd.edu.ph,cmfestin@dcs.upd.edu.ph
}

\begin{abstract}
Load-aware routing metrics aim to address congestion and load-balancing issues in wireless mesh networks by directing traffic either around or away from loaded regions of the network. However, the fact that load estimates used to choose routes are in turn affected by resulting routing changes in a cyclical manner makes it difficult to preset the sensitivity of route adaptation to load. On the other hand, desensitizing nodes to traffic load may render them unresponsive, leading to inaccuracies in load estimates and difficulties in achieving proper traffic distribution. To address these issues, we present CLAW, a novel channel load-aware routing metric that handles the routing process in a manner analogous to a feedback control system. Simulations show that CLAW significantly improves network performance in both throughput and delay against hop count and other competing load-aware routing metrics found in the literature.
\end{abstract}

Keywords - Channel load aware, load adaptive, routing metric, wireless mesh networks.

\section{InTRODUCTION}

Wireless mesh networks (WMNs) are communication networks composed of wireless nodes that participate either as routers or clients of the network [1]. The mesh routers are generally static or minimally mobile and serve either as dedicated forwarding nodes and access points for clients like desktop PCs, laptops and mobile devices, or both. Collectively, mesh routers form the backbone of the wireless network which enables traffic to be transported and ensures reachability between participating nodes [2].

In recent years, the use of WMNs has become an attractive strategy for the rapid, flexible and low-cost deployment of wireless services for a wide variety of applications including broadband home networking and automation [3], community mesh networking [4], public safety and disaster ad hoc communication scenarios [5], and medical applications [6]. Despite this attractiveness however, several deployment challenges remain, such as problems of interference and contention, load imbalance, and congestion [1]. As these problems become more significant, network throughput and delays inevitably degrade.

To address this issue, load-aware routing metrics have been developed so that flows may avoid congested regions of the network [7]. However, making routes adapt to load changes does not necessarily improve performance because the load estimates used to choose routes are themselves affected by the route choices with feedback effects that cause routing instabilities such as oscillation [8]. To address this, we developed a novel channel load aware (CLAW) routing metric that handles the routing process in a manner analogous to a feedback control system. Our simulations show that CLAW is able to stabilize routes, significantly improving network performance in both throughput and delay against conventional hop count metrics, as well as other competing load aware routing metrics found in the literature.

This paper traces the evolution of CLAW's design, and hopes to share our findings from its evaluation. In Section II, we present a broad view of the issues in load-aware and loadadaptive routing. Section III describes our early attempt to introduce load-awareness in routing through CLM, a loadaware metric with a novel load-sensing technique, and discusses stability issues we have uncovered. Section IV introduces CLAW, our subsequent approach which integrates stability control with CLM's load-sensing technique. Section $\mathrm{V}$ reports on CLAW's behavior and performance in simulations. Finally, Section VI summarizes our key findings, and maps the road ahead.

\section{Issues in Load-Aware Routing}

In load-aware routing, the load estimates used to choose routes are themselves affected by the route choices with feedback effects that cause instability [9]. One way to address this issue is to make nodes relatively unresponsive to the traffic they themselves induce [7]. However, this approach results in inaccurate channel load estimation, leading to an inability to achieve load distribution and balancing [10]. In addition, these approaches often require changes in either the MAC or network layers in the form of packet flow monitoring, additional inter-node information exchange, and complex route computations.

Indeed, on-demand routing protocols may be used instead of proactive ones [11]. In theory, on-demand routing protocols do not recompute routes between source-destination node pairs unless there is link breakage [12], thus avoiding loadtriggered recomputations that may lead to oscillations. However, interference, noise or channel fading [13] may result in disconnections that may still trigger such recomputations. Furthermore, the supposed stability achieved with such protocols comes at the price of reduced sensitivity to congestion events.

The above-mentioned solutions are designed to control routing oscillations only. However, these do not address other 
forms of routing instability such as the inability of the routing algorithm to quickly converge, or its tendency to build routes with loops due to the presence of zero or near-zero load metric values. These issues are discussed in the next section, where we present our early attempt to build a load-aware metric called CLM.

\section{CLM: An Early Attempt at Load-Awareness}

In this section we describe our early investigations in the use of load-aware routing metrics. First we describe the design of the metric itself, and then we discuss the results of our evaluation of this early design.

\section{A. Load Metric}

From our previous work [14], we consider the channel as busy when the node involves itself in transmission or reception of packets within the channel, or it senses any energy including those that hinders successful transmission such as those resulting from collisions, interference, or other forms of noise. The channel may likewise be considered busy when the node is blocked from accessing the channel, such as due to the back-off and defer periods in the distributed coordination function (DCF) in the IEEE 802.11 standard [15]. If all these events can be classified into one of two fractional components of time, called $T_{\text {sensedEnergy }}$ and TblockedForAccess, then the channel load is the total fraction of time that a node is busy due to any of these contributing events. Equation (1) expresses this definition of channel load, where CLM means channel load metric.

$$
C L M=\left\{T_{\text {sensedEnergy }} U T_{\text {blockedForAccess }}\right\}
$$

\section{B. Evaluating CLM}

Our evaluation of CLM consisted of simulation experiments in Network Simulator 2 (ns2) [16] with the OLSR extension as used in [17]. These experiments were aimed at testing whether our metric would result in routes that (1) avoided busy regions of the network, (2) achieved load distribution, and (3) achieved better throughput compared to the commonly used hop count [12] metrics. The experiment used a mesh network composed of 81 nodes uniformly distributed in a $1160 \times 1160$ square meter area (Figure 1). Each node used $802.11 \mathrm{~b}$ wireless device with nominal bit rate of $1 \mathrm{Mbps}$. The main traffic flow (from node 0 to node 80) sent $100 \mathrm{kbps}$ of constant bit rate (CBR) traffic over UDP with a packet size of 1000 bytes. An interfering traffic set between node 40 and 48 should veer the path of the main flow away from the center. This traffic between node 40 and 48 varied from $0,50,100,150,200,300,400,450$, until $500 \mathrm{kbps}$.

Figures 2 and 3 show the result of the simulations. Although CLM-based routing veered the main traffic away from the loaded center region, in most of the scenarios it was not better than hop count in terms of throughput and packet delay. The worst scenario occurred when there was no interfering traffic at the center of the mesh network. In this scenario, on the average, the received throughput achieved by CLM-based routing was $37 \%$ less and the packet delay was $52 \%$ longer than those achieved in hop count based routing.
This performance degradation has been reported due to oscillation alone [7], [10], [11], however our simulations showed that this is rather due to the inability of the routing algorithm to converge or to its tendency to build routing loops.

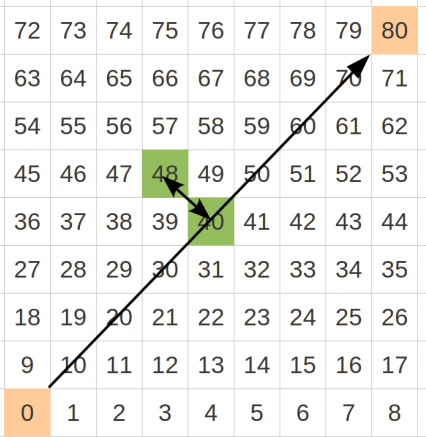

Figure 1: A WMN with node 0 sending traffic to node 80 , while nodes 48 and 49 congest the center.

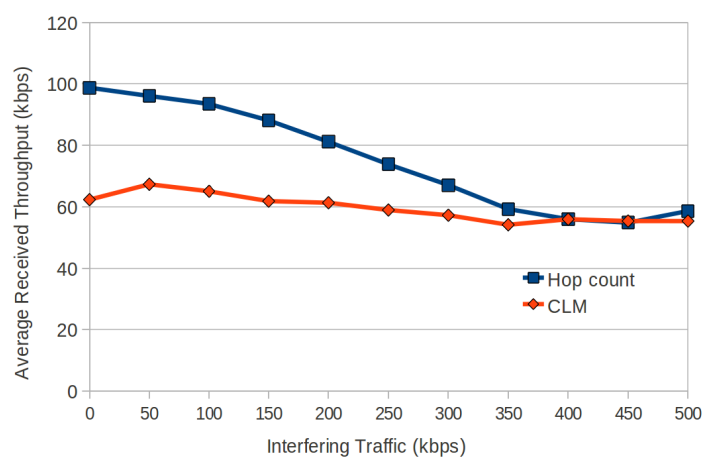

Figure 2: Throughput comparison between hop count and CLM

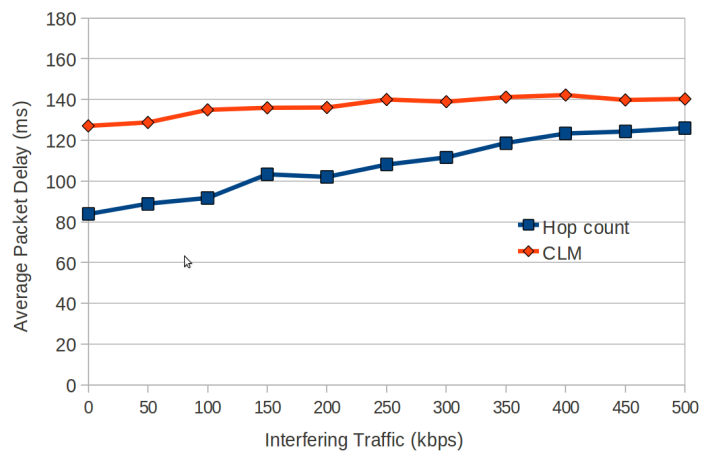

Figure 3: Packet-delay comparison between hop count and CLM

\section{Routing Stability Issues in CLM}

Our subsequent analysis of simulation traces revealed that CLM's instability was not primarily caused by oscillations, but is instead due to its inability to quickly converge and its tendency to build routing loops. This behavior is caused by the zero or near-zero load weights for idle regions. When a node sends traffic towards a particular destination, the load of the regions along the path get load weights that are significantly greater than zero. Consequently, the idle regions become better paths because of their zero or near-zero weights. The path cost through idle regions remains better, even if the route 
goes back and forth in a loop within a set of nodes, than the cost of the path where the current traffic is traversing. In turn, more packets accrue in transit across the mesh network causing more contention and collision of packets, and packets reaching their time-to-live (TTL) limits. As a result more packets are dropped or are not able reach their destination decreasing throughput significantly. The longer packet delay, on the other hand, is due to high rate of packets dropped because of collisions mentioned above and to the longer path taken by other packets because of route oscillations .

\section{CLAW: CLM with Stability Control}

A load adaptive routing network can be considered as a system analogous to a control system with a feedback control [9]. Figure 4 illustrates the system block diagram for such a system. We re-designed CLM by integrating its load-sensing technique as described in Equation (1) with a mechanism that addresses non-convergence of the routing algorithm at light load scenarios. This mechanism is represented by the nonadaptive area in the weight mapping function in Figure 4 which effectively serves as a bias [8]. To keep the control method simple, the re-design did not include changes in the routing algorithm and route update blocks, but rather focused on the channel load weighing function and on an added exponential weighted averaging function. The latter exponential weighted averaging block regulates further instability contributed by sudden traffic bursts, and is defined in Equation (3).

\section{Evaluation and Results}

Using the simulation set up described in Section III, we present the behavior and performance of a CLAW-based routing, both in two sets of contrasting traffic scenarios. The first set of scenarios had a single source of traffic towards the other end of the network. An interfering traffic was present at the center of the network, along the path of the main traffic. The mesh network is widely open in terms of routing and propagating packets. In this kind of situation, a load-adaptive routing has the tendency to oscillate the worst and become unstable because of the availability of more lightly-loaded routes to choose from.

In the second set of scenarios, there were five randomly chosen traffic sources creating cramped situations for routing packets. CLAW was pitted against metrics based on hop counts, wireless interface buffer queue (IFQ), and contention window (CW). In the literature [7], [18], the IFQ and CW window have been extensively used as the metric for accounting channel load. Accordingly, we derive loadadaptive routing metrics based on IFQ and CW called $Q u A W$ and $C O A W$ respectively. We equipped these metrics with stability control similar to that of CLAW (Section IV.)

\section{A. CLAW Better Congestion Avoidance Mechanism}

Figure 5 summarizes the average received throughput of CLAW-, CLM-, and hop count-based routing for the first set of simulation scenarios. With the stability control of CLAW, it improves on CLM performance outperforming that of hop count. By avoiding the center area as the center traffic gets heavier, received throughput for CLAW is, on the average, $25 \%$ better than hop count. At the heaviest center traffic, CLAW's received throughput is $64 \%$ more.

However, our simulations showed that CLAW-based routing has not totally eliminated routing oscillations. This result is of course due to routes adapting to load changes. Nevertheless, CLAW's minimum weight made routing loops costly. This stabilized routes to a certain degree. The achieved stability and the ability to veer away from loaded regions of the network improved received throughput in comparison to the hop count metric.

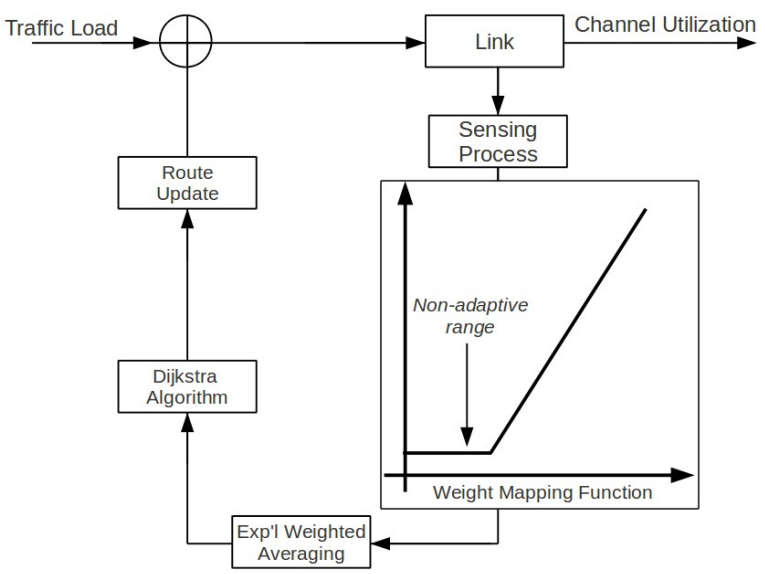

Figure 4: The feedback system block diagram for CLAW

$$
\begin{gathered}
C L M_{\text {weight }}=\left\{\begin{array}{l}
\min _{\text {weight }} \\
C L M \quad \text { otherwise }
\end{array}\right. \\
C L A W(t)=[1-\alpha] C L A W(t-1)+\alpha C L M_{\text {weight }}
\end{gathered}
$$

Table 1. The Parameters Used in the Stability Control of ClaW.

\begin{tabular}{|c|c|c|}
\hline Parameter & Meaning & Value \\
\hline $\begin{array}{c}\text { Sensing } \\
\text { Interval }\end{array}$ & Interval in-between probes to sense busy state & $1 \mathrm{~ms}$ \\
\hline $\begin{array}{c}\text { Observation } \\
\text { period }\end{array}$ & $\begin{array}{c}\text { The period of time in which the sensed values are } \\
\text { averaged into the CLM value }\end{array}$ & $2 \mathrm{~s}$ \\
\hline min weight & $\begin{array}{c}\text { The damping value that represents the non-adaptive } \\
\text { area in the weight mapping function }\end{array}$ & 0.1 \\
\hline max weight & $\begin{array}{c}\text { The maximum weight value for CLA. Represents } \\
100 \% \text { load }\end{array}$ & 1 \\
\hline$\alpha$ & $\begin{array}{c}\text { Coefficient used in the exponential weighted } \\
\text { average; used to clip sudden changes in channel } \\
\text { load }\end{array}$ & 0.5 \\
\hline
\end{tabular}

\section{B. CLAW Better Load Awareness Capability}

For the second set of simulation scenarios, Figures 6 and 7 summarize the results. On the average, CLAW's received rate is $14 \%$ better than hop count and $22 \%$ at the most with slightly better packet delay. QuAW behaves almost similar to hop count because simulation traces show that the queue level of a node increases only when the node concerned continues 
to pump traffic into an already saturated channel. In the absence of saturated channel, queue level remains near the zero level. With the minimum weight (bias) set, QuAW-based routing behaved similar to hop count.

On the other hand, CoAW's received throughput is on the average $6 \%$ better than hop count and $11 \%$ at its best. This better throughput is due to its capability to sense contention from traffic flowing along adjacent paths. Consequently, it can direct packets through regions with less contention resulting to better performance than hop count metric. However, it still performs less than CLAW because when neighboring nodes do not compete for use of the channel, i.e only one node pumps traffic into the channel, it can hardly sense channel load. Hence, QuAW is not able to avoid loaded regions of the network better than CLAW, resulting to an inferior performance than CLAW.

\section{Conclusions and Future Work}

Naively replacing commonly-used hop count routing metrics [12] with a load-aware ones would not necessarily improve network performance because of feedback effects that cause route instability. In WMNs, this instability has been described primarily as oscillations [7], [10], [11]. Our investigations, however, revealed that performance degradations may also be due to route convergence issues. To the best of our knowledge, this phenomenon has not yet been reported in the literature.

To address stability issues in load-adaptive routing without sacrificing the accuracy of channel load estimates, we developed a new metric called CLAW (channel load aware routing metric). CLAW treats the routing process in a manner analogous to a control system with feedback control. It can stabilize routes without sacrificing sensitivity to load changes. Simulations in different traffic scenarios showed that CLAW improved network performance in terms of throughput and delay against hop count and competing load aware routing metrics in the literature.

Despite the level of stability achieved and network performance improvement attained by CLAW-based routing, it still exhibits some degree of routing oscillation. It would be interesting to conduct a deeper investigation into the adjustable parameters of the feedback control for CLAW and an exploration of other control methods like adaptive control [19] and fuzzy logic [20] in the future. Other forwarding schemes that may not be prone to loops such as those based on source routing [21] are also worth exploring. Ultimately, the usefulness of this metric can only be fully realized through actual, working implementations, rather than through theoretical simulations. All of these undertakings are hopefully addressed as part of a future work.

\section{AcKNowledgment}

This work has been supported by the Engineering Research and Development for Technology (ERDT) Consortium, Department of Science and Technology - Science Education Institute (DOST-SEI), Philippines.

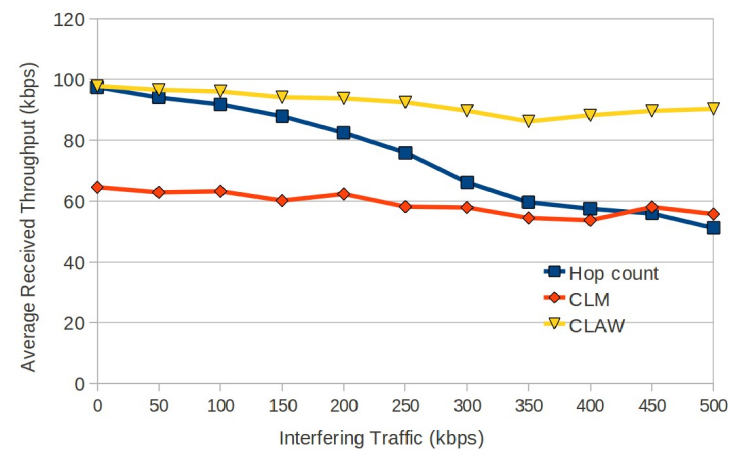

Figure 5: Average Received Throughput Comparison of Hop Count, CLM, and CLAW

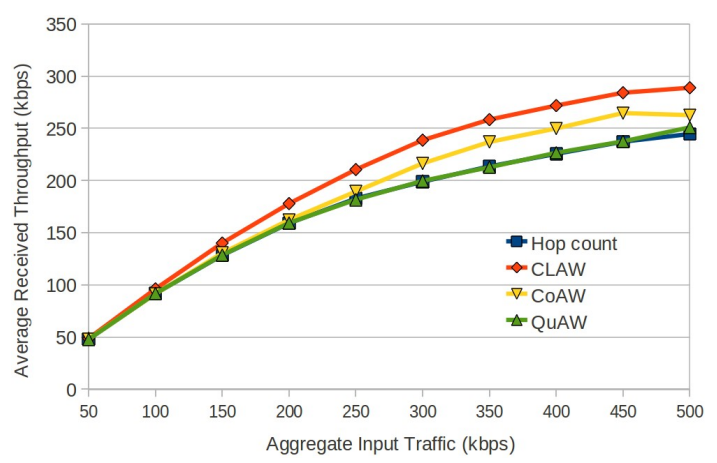

Figure 6: Average Received Throughput Comparison Between Hop Count, CLAW, CoAW, and QuAW

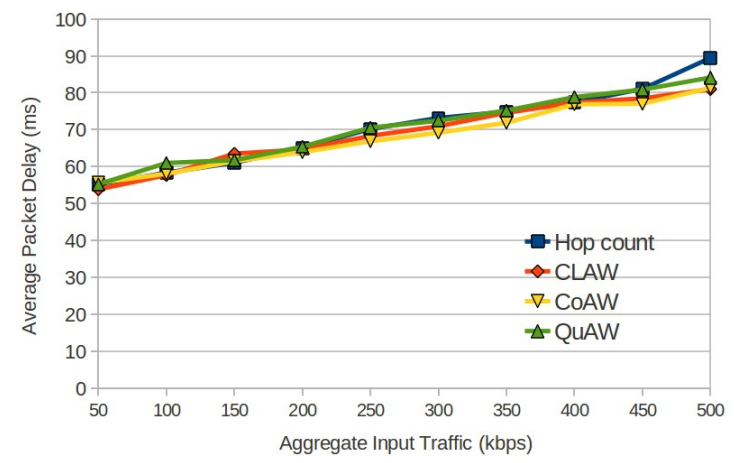

Figure 7: Average Packet Delay Comparison Between Hop Count, CLAW, CoAW, and QuAW

\section{REFERENCES}

[1] I. F. Akyildiz, X. Wang, and W. Wang, "Wireless mesh networks: a survey," Comput Netw ISDN Syst, vol. 47, no. 4, pp. 445-487, 2005.

[2] J. Eriksson, S. Agarwal, P. Bahl, and J. Padhye, "Feasibility study of mesh networks for all-wireless offices," in Mobisys 2006, New York, NY, USA, 2006, pp. 69-82.

[3] E. Callaway, P. Gorday, L. Hester, J. A. Gutierrez, M. Naeve, B. Heile, and V. Bahl, "Home networking with IEEE 802.15. 4: a developing standard for low-rate wireless personal area networks," Commun. Mag. IEEE, vol. 40, no. 8, pp. 70-77, 2002.

[4] Carol Ellison, "Municipal Broadband: A Potential twenty-first century utility," N. Y. Univ. J. Legis. Public Policy, 2008.

[5] K. Kanchanasut, A. Tunpan, M. Awal, T. Wongsaardsakul, D. Das, and Y. Tsuchimoto, "Building A Long-distance Multimedia Wireless Mesh Network for Collaborative Disaster Emergency Responses Operation in Disaster-affected Areas," Internet Education and Research Laboratory, Asian Institute of Technology, Thailand, Apr. 2007. 
[6] T. Trimble, R. Mishra, B. S. Manoj, R. Rao, L. Lenert, and B. Braunstein, "Feasibility of using distributed Wireless Mesh Networks for medical emergency response.," in AMIA 2006, vol. 2006, pp. 86-90.

[7] L. T. Nguyen, R. Beuran, and Y. Shinoda, "An interference and load aware routing metric for Wireless Mesh Networks," Int. J. Ad Hoc Ubiquitous Comput., vol. 7, pp. 25-37, Dec. 2011.

[8] D. Bertsekas, "Dynamic behavior of shortest path routing algorithms for communication networks," IEEE Trans. Autom. Control, vol. 27, no. 1, pp. $60-74$, Feb. 1982

[9] Hao Wang and M. R. Ito, "Dynamics of load-sensitive adaptive routing," in ICC 2005, 2005, vol. 1, pp. 213-217 Vol. 1.

[10] H. Badis, I. Gawedzki, and K. Al Agha, "QoS routing in ad hoc networks using QOLSR with no need of explicit reservation," in VTC 2004-Fall, 2004, vol. 4, pp. 2654 - 2658 Vol. 4.

[11] M. Catalan-Cid, J. L. Ferrer, C. Gomez, and J. Paradells, "Contentionand interference-aware flow-based routing in wireless mesh networks: design and evaluation of a novel routing metric," EURASIP $J$ Wirel Commun Netw, vol. 2010, pp. 106:1-106:20, Apr. 2010.

[12] C. Perkins, E. Belding-Royer, and S. Das, "Ad hoc On-demand Distance Vector (AODV) Routing, rfc 4561.” IETF, Jul-2003.

[13] Rappaport, Wireless Communications: Principles and Practice. Pearson Education, 2009

[14] N. A. Macabale Jr, R.M. Ocampo, and C. M. Festin, "Congestion-free Routes for Wireless Mesh Networks," in ISMAC 2011, Hokkaido, Japan, 2011.

[15] IEEE, "802.11: Wireless LAN Medium Access Control (MAC) and Physical Layer (PHY) Specifications- Revision of the 802.11-1999 standard," 2007.

[16] S. McCanne and S. Floyd, "The Network Simulator 2 - NS2," 21-Jul2009. [Online]. Available: http://www.isi.edu/nsnam/ns/. [Accessed: 21-Jul-2009].

[17] W. Cordeiro, E. Aguiar, W. Moreira, A. Abelem, and M. Stanton, "Providing Quality of Service for Mesh Networks Using Link Delay Measurements," in ICCCN 2007, 2007, pp. 991-996.

[18] Mahammad Siraj and Kamalrulnizam Abu Bakar, "A load Balancing Interference Aware Routing Metric (LBIARM) for Multi-HOP Wireless Mesh Network," Int. J. Phys. Sci., vol. 7(3), p. 45+-461, Jan. 2012.

[19] K. J. Astrom and D. B. Wittenmark, Adaptive Control: Second Edition, 2nd ed. Dover Publications, 2008.
[20] J. Jantzen, Foundations of Fuzzy Control, 1st ed. Wiley, 2007.

[21] D. A. Maltz and D. B. Johnson, "The Dynamic Source Routing Protocol (DSR) for Mobile Ad Hoc Networks for IPv4, rfc 4728.” IETF, 2007.

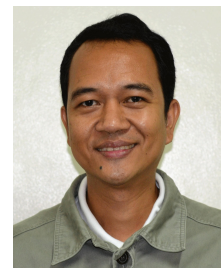

Nemesio A. Macabale Jr. received his B.S. degree in electronics and communications engineering from the University of Santo Tomas, Manila, Philippines in 1993, his Master of Computer Science at the De la Salle University, Manila, Philippines in 2001, and his Ph.D. in electrical and electronics engineering from the University of the Philippines, Quezon City, Philippines in 2013. He is currently an associate professor at the Department of Information Technology and the Director of the Information Systems Institute, Central Luzon State University, Science City of Munoz, Philippines.

Roel M. Ocampo received his B.S. degree in electrical engineering from the University of the Philippines, Diliman, Quezon City, Philippines in 1990, and his $\mathrm{Ph} . \mathrm{D}$. in electrical and electronic engineering from the University College London, London, U.K. in 2007. He is currently an Associate Professor at the Electrical and Electronics Engineering Institute, University of the Philippines, Diliman, Quezon City.

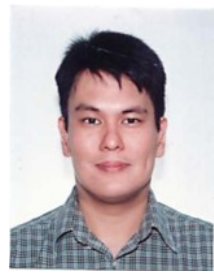

Cedric Angelo M. Festin is an Associate Professor at the Department of Computer Science, College of Engineering, University of the Philippines. He is head of the Network and Distributed Systems Group and and the current chairperson of the department. He has a Ph.D. from University College London, and a Master's Degree in Electrical Engineering and an undergraduate degree in Computer Science from the University of the Philippines. Recently, he was a Visiting Researcher at the University

of California Berkeley, working with the Technology and Infrastructure for Emerging Regions (TIER) group. His research areas is in the design, development, and benchmarking of network protocols for applications for emerging regions, such as those used for e-Learning and health information systems. 